\title{
Effective and Safe Treatment of Psoriatic Disease with the Anti-IL-23p19 Biologic Tildrakizumab: Results of a Real-World Prospective Cohort Study in Nonselected Patients
}

\author{
Katharina A. Drerup Claudia Seemann Sascha Gerdes Ulrich Mrowietz \\ Psoriasis-Center at the Department of Dermatology, University Medical Center Schleswig-Holstein, \\ Campus Kiel, Kiel, Germany
}

\section{Keywords}

Psoriasis · Treatment · Tildrakizumab · IL-23 · Real-world therapy

\begin{abstract}
Background: After registration of drugs, evidence about efficacy and safety is solely based on data of phase $2 / 3$ clinical trial programs. A major drawback is the selection of patients following inclusion/exclusion criteria. There is a considerable time and knowledge gap between study and registry data that evaluate real-world evidence (RWE). To close this gap, prospective cohort data are helpful. Objectives: Soon after tildrakizumab, an interleukin 23p19-inhibitor, was registered for moderate-to-severe plaque psoriasis, a prospective single-center cohort study was established to evaluate efficacy and safety of tildrakizumab in daily practice. Methods: Following approval of tildrakizumab, patients with moderate-to-severe plaque psoriasis eligible for systemic treatment were included into the Kiel Tildra Cohort (KTC) and followed using routine assessments of efficacy, psoriasis area and severity index (PASI), body surface area (BSA), dermatology life quality index (DLQI), itch (visual analog scale), and safety. Data of the KTC were compared to the respective phase 3 clinical trials. Results: The KTC included 150 patients
\end{abstract}

karger@karger.com www.karger.com/drm

Karger $\stackrel{\text { ' }}{5}$

GOPEN ACCESS
(C) 2021 The Author(s)

Published by S. Karger AG, Basel

This is an Open Access article licensed under the Creative Commons Attribution-NonCommercial-4.0 International License (CC BY-NC) (http://www.karger.com/Services/OpenAccessLicense), applicable to the online version of the article only. Usage and distribution for commercial purposes requires written permission. differing substantially from those in the trial program. There was a high rate of previous systemic (87.3\%) and biologic (31.8\%) therapy and of comorbidity in the KTC as compared to the phase 3 studies. Due to the best practice approach, baseline PASI was lower in the KTC, but DLQI was similar in both groups. At the time of this analysis, 126 patients completed week 28, 92 patients week 52, and 58 patients week 76 , respectively. There was a constant improvement in PASI, $\mathrm{BSA}, \mathrm{DLQI}$, and itch from baseline until week 76 . There was no clinically meaningful laboratory abnormality. Conclusions: Patients treated in routine practice with tildrakizumab differed substantially from the phase 3 studies. Despite systemic pre-treatment and increased comorbidity, tildrakizumab showed comparable efficacy and safety in the KTC. Prospective cohort studies are a suitable tool to generate RWE before registry data become available.

(C) 2021 The Author(s).

Published by S. Karger AG, Basel

\section{Introduction}

Effective and safe long-term disease control is today's paradigm to treat psoriatic disease, a frequent chronic illness for which there is no cure. Substantial progress has been made in recent years in the development of drugs for 
Table 1. Demographics of patients included into the KTC in comparison to those recruited during the phase 3 study (reSURFACE)

\begin{tabular}{lll}
\hline & KTC & $\begin{array}{l}\text { reSURFACE } \\
(100 \mathrm{mg})\end{array}$ \\
\hline Male & & $72 \%$ \\
Age, years & $64.7 \%$ & $44.6( \pm 13.6)$ \\
Age range, years & $47.6( \pm 17.2)$ & $19-80$ \\
Weight, kg & $20-88$ & $89.35( \pm 22.12)$ \\
Body mass index, $\mathrm{kg} / \mathrm{m}^{2}$ & $89.2( \pm 21.3)$ & $\mathrm{na}$ \\
Previously treated with nonbiologic systemic therapy (excluding phototherapy) & $28.3( \pm 6.1)$ & $31.8 \%$ \\
Previously treated with biologics & $24.7 \%$ & $17.9 \%$ \\
Family predisposition, yes/no/na & $38 \% / 37 \% / 25 \%$ & na \\
Smoker/ex-smoker/non-smoker, yes/no/na & $45 \% / 32 \% / 23 \%$ & na \\
Age at onset of psoriasis, years & $28.6( \pm 20.5)$ & na \\
\hline
\end{tabular}

KTC, Kiel Tildra Cohort; na, no data.

this purpose. The majority of people with psoriatic disease present with skin lesions only, and if they qualify for systemic therapy, the group of IL-23p19 antagonists may be the biologic class of choice. In most countries, 3 therapeutic antibodies of this group are registered for first-line treatment: guselkumab [1,2], risankizumab [3], and tildrakizumab [4]. The latter two are administered every 12 weeks during maintenance therapy, which is helpful regarding management and safety monitoring according to guidelines. Treatment adherence seems favorable with long injection intervals.

When drugs are newly available for prescription, data about efficacy and safety are only based on clinical trials and rarely data about treatment times of more than 1 year. There is a considerable gap in time until registry data are published based on nonselected patients. It has been shown from registry data analysis that only $78.4 \%$ of patients presenting to dermatology offices and departments are eligible for clinical trials [5]. In particular, extensive comorbidity and failure of previous therapies often present a therapeutic challenge [6].

To close this gap, prospective cohort studies in nonselected patients initiated soon after market access of new drugs are helpful.

In the clinical trials, tildrakizumab proved to be effective and safe for the treatment of plaque psoriasis, and soon after registration, results of an open-label extension over 3 years were published. To prove that in nonselected patients tildrakizumab provided the same benefit, we started a prospective cohort study in 150 patients with plaque psoriasis consecutively recruited when presenting to our psoriasis center. The study remained an academic investigation (Kiel Tildra Cohort [KTC]) and was not sponsored. Here, we present the first data after more than half of the patients completed 52 weeks of tildrakizumab therapy.

\section{Methods}

\section{Patients and Study Design}

The study was approved by the Ethics Committee of the University of Kiel (AZ: D425/20). Before inclusion into the study, patients gave their informed consent.

Recruitment started in December 2018, soon after tildrakizumab was launched in Germany. Patients presenting at the psoriasis center for routine care were recruited consecutively. Inclusion criteria were the need for systemic biologic therapy in patients qualifying for treatment with an IL-23p19 inhibitor. As per label, patients with moderate-to-severe psoriasis fulfilling the criteria published by the European Consensus, including the use of upgrade criteria, were selected. All patients were screened for latent tuberculosis using interferon gamma release assay and chest X-ray as recommended in the summary of medicinal product characteristics and by local guidelines. Tildrakizumab was given as per label with a dose of $100 \mathrm{mg}$ at baseline, after 1 month, and thereafter every 3 months. The drug was always given to the patient by the dermatologist or trained study nurses at the site. In none of the patients was the dose increased to $200 \mathrm{mg}$, which is possible in case of insufficient response.

The following assessments were routinely performed at baseline and at each following visit: Psoriasis Area and Severity Index (PASI), body surface area (BSA), patient global assessment (PaGA, scale from 0 to 4 ), itch visual analog scale (VAS), and dermatology life quality index (DLQI).

Safety monitoring was performed at baseline and thereafter in 3-month intervals before the drug was given to the patient. This included laboratory controls (hematology, GOT, GPT, gamma glutamyl transferase, creatinine, C-reactive protein), inspection of the entire skin, and an interview about tolerability. 


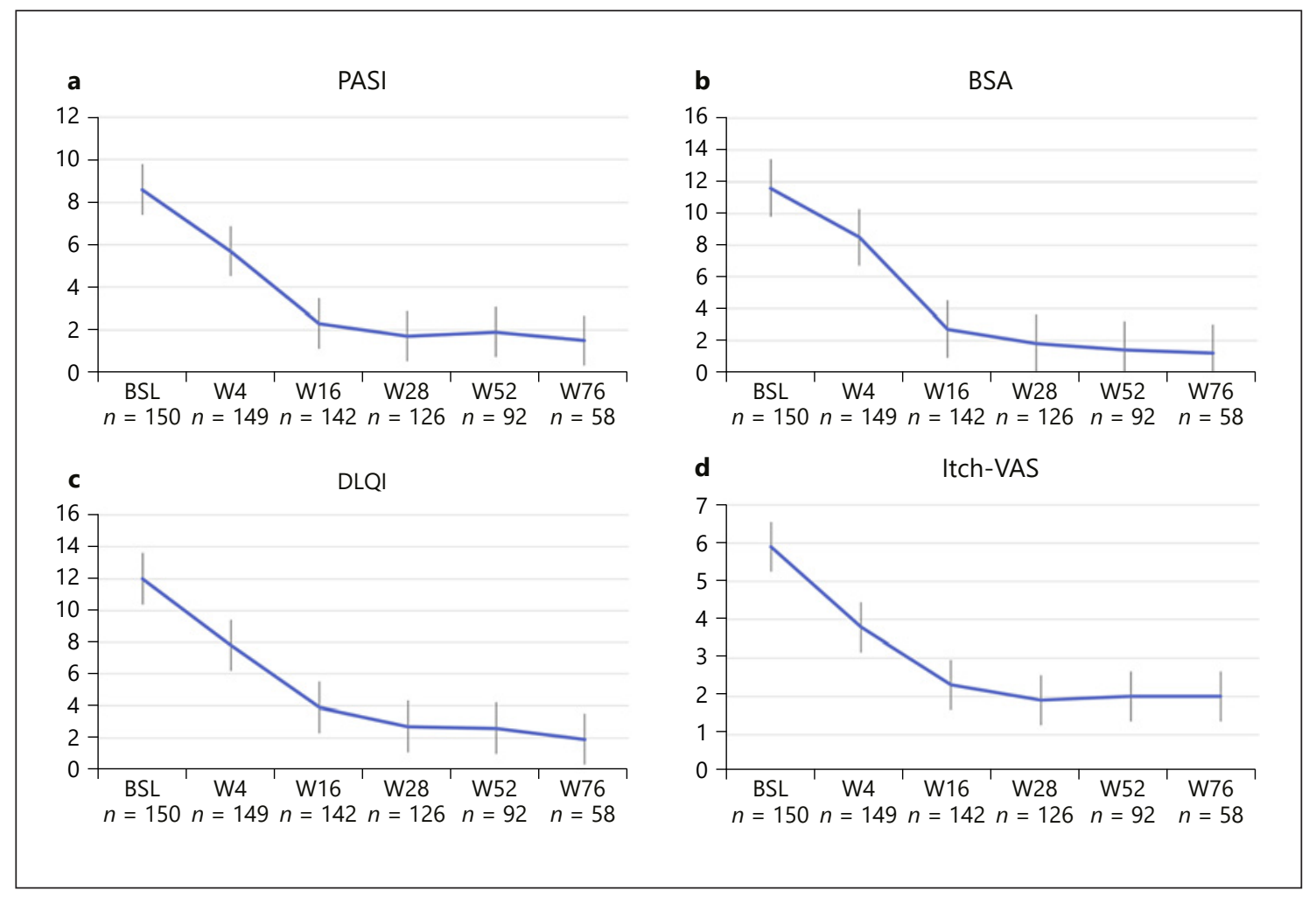

Fig. 1. Course of PASI (a), BSA (b), DLQI (c), and itch-VAS (d) in patients included into the KTC from baseline until week 76. Values are mean and standard deviation.

The results of the KTC were compared to the data of the phase 3 trial of tildrakizumab (reSURFACE 1 and 2).

\section{Results}

Until the timepoint of this first analysis (August 20, 2021), 150 patients with plaque psoriasis were included in the study. Demographics of the patients are shown in Table 1 .

Patients in the KTC were thoroughly screened for associated conditions. The most frequently reported comorbidity was hypertension (33\% in the KTC, $25 \%$ in the reSURFACE study with $100 \mathrm{mg}$ tildrakizumab, respectively). This was followed by depression (18\% in the KTC, no data in the reSURFACE study), diabetes mellitus type 2 ( $10 \%$ vs. $3 \%$ ), and hyperlipidaemia ( $8 \%$ vs. $6 \%$ ). Baseline assessments of patients are as shown in Table 2.

At the timepoint of this first report, 126 of the $150 \mathrm{pa}-$ tients have already completed week 28 (4 injections) of treatment with tildrakizumab, 92 have completed 52 weeks (6 injections), and 58 have been followed up to 76
Table 2. Baseline assessments of the KTC in comparison to patients recruited during the phase 3 study (reSURFACE) at week 28

\begin{tabular}{lll}
\hline Assessment & $\begin{array}{l}\text { KTC } \\
(n=150)\end{array}$ & $\begin{array}{l}\text { reSURFACE } \\
(100 \mathrm{mg})(n=328)\end{array}$ \\
\hline PASI & $\begin{array}{l}8.6 \pm 4.2 \\
11.6 \pm 9.1 \%\end{array}$ & 20.5 \\
BSA & $12.0 \pm 5.6$ & $34.2 \%$ \\
DLQI & $5.9 \pm 2.6$ & 14.8 \\
Itch-VAS & na \\
\hline
\end{tabular}

KTC, Kiel Tildra Cohort; na, no data.

Table 3. Absolute PASI achieved in the KTC until week 76

\begin{tabular}{llll}
\hline Absolute PASI & $\begin{array}{l}\text { Week 28 } \\
(n=126)\end{array}$ & $\begin{array}{l}\text { Week 52 } \\
(n=92)\end{array}$ & $\begin{array}{l}\text { Week 76 } \\
(n=58)\end{array}$ \\
\hline$\leq 3$ & $81 \%$ & $71 \%$ & $80 \%$ \\
$\leq 1$ & $42 \%$ & $39 \%$ & $39 \%$ \\
\hline
\end{tabular}


weeks (8 injections). Figure 1 shows the course of PASI, BSA, DLQI, and itch-VAS over 76 weeks in the KTC. A continuous improvement of all 4 assessments can be seen over time.

Table 3 shows the improvement of psoriasis as assessed by absolute PASI $(<3$ and $<1)$ as this approach seems more meaningful particularly when the baseline PASI is low. Related to the safety monitoring that was routinely performed before each injection, no treatmentrelated adverse event was seen.

At the timepoint of this analysis, 13 patients (8.7\%) had dropped out of the cohort. Three patients were switched to another treatment after 2 doses of tildrakizumab ( 1 patient due to the diagnosis of rosacea made by an external dermatologist who regarded this as a side effect of tildrakizumab therapy and 2 patients because of fatigue or gastrointestinal side effects they attributed to tildrakizumab treatment), and 2 other patients were switched to another medication due to primary nonresponse. In 5 other patients, the treatment was also discontinued after 5 injections because of secondary nonresponse. One patient preferred oral therapy with tablets after 3 injections. Two other patients did not show up after the first and third application and could not be contacted.

\section{Discussion}

Although necessary for registration of new drugs, clinical trials and respective data are hampered by the fact that patients are highly selected due to stringent inclusion and exclusion criteria [5]. In particular, patients with extensive pretreatment are excluded as these patients need to wait until their psoriasis is worse until meeting the common cutoff of PASI $>12$. In clinical practice, this is not possible. This is reflected by the mean baseline PASI of $8.6 \pm 4.2$ of the patients included in the KTC in comparison to the PASI of the reSURFACE studies $(20.5 \pm 7.63)$. There were no washout periods in the KTC study. Treatment with tildrakizumab was started immediately or only a few weeks after discontinuation of the previous treatment. Frustration about insufficient prior treatment and/or adverse effects is reflected by the baseline DLQI of the KTC that is similar to the reSURFACE study.

Patients in the KTC showed a significantly increased rate of pretreatment as compared to the reSURFACE studies. This was most pronounced for conventional systemics but also for biologic agents.
Treatment with tildrakizumab led to a rapid decrease in disease severity (according to PASI and physicians' global assessment), a decrease in itch, and an improvement of health-related quality of life (DLQI).

When data regarding absolute PASI improvement were analyzed, patients of the KTC achieved the same level of improvement as the selected patients in the reSURFACE trials. In the light of the major differences between the KTC and the reSURFACE studies regarding pretreatment and the number of associated diseases, tildrakizumab showed an excellent performance in this difficult-totreat patient population.

There was a low rate $(8.7 \%)$ of patients that terminated the treatment until the date of analysis. In particular in patients with a relatively low PASI at baseline but a need for treatment, it is important to determine the therapeutic efficacy by using absolute rather than relative PASI improvement. At the baseline visit, 4 of the 150 patients had a PASI $<3$. In the KTC, more than $81.0 \%$ of patients showed a PASI $<3$ after 28 weeks and more than $42 \%$ a PASI $<1$ that is indicative of clear skin. This is reflected by the corresponding low DLQI substantiating that tildrakizumab led to a high treatment satisfaction: $61 \%$ of patients showed a DLQI of 1 or 0 after 28 weeks.

After 1 year (week 52) of treatment, $71 \%$ achieved a PASI $<3$ and $39 \%$ a PASI $<1$; a DLQI of 0 or 1 was reported by $52 \%$. This trend continued until week 76 ; however, these data are based on a smaller number of patients yet.

In none of the patients of the KTC analyzed so far did laboratory parameters show changes that led to a medical action or decision. In the light of the substantial comorbidity of the KTC patients, this adds to the favorable benefit-risk assessment of tildrakizumab.

Registry data published so far have demonstrated that the IL-12/23p40 inhibitor ustekinumab always had the best drug survival. It can be assumed that IL-23p19 antagonists such as tildrakizumab may have similar characteristics. The first data of the KTC points in this direction.

\section{Key Message}

Tildrakizumab is highly effective in real-world psoriasis pretreated patients.

\section{Statement of Ethics}

The study was approved by the Ethics Committee of the University of Kiel (AZ: D425/20). All patients provided written informed consent before inclusion into the study. 


\section{Conflict of Interest Statement}

Ulrich Mrowietz has been an advisor and/or received speaker's honoraria and/or received grants and/or participated in clinical trials of the following companies: AbbVie, Aditxt, Almirall, Amgen, Aristea, Boehringer-Ingelheim, Bristol-Myers Squibb, Celgene, Dr. Reddy's, Eli Lilly, Foamix, Formycon, Immunic, Janssen, LEO Pharma, Medac, MetrioPharm, Novartis, Phi-Stone, Pierre Fabre, Sanofi-Aventis, UCB Pharma.

Sascha Gerdes has been an advisor and/or received speaker's honoraria and/or received grants and/or participated in clinical trials of the following companies: AbbVie, Affibody AB, Akari Therapeutics Plc, Almirall-Hermal, Amgen, Anaptys Bio, AstraZeneca AB, Biogen Idec, Bioskin, Boehringer-Ingelheim, Celgene, Dermira, Eli Lilly, Foamix, Forward Pharma, Galderma, Hexal AG, Incyte Inc., Janssen-Cilag, Johnson \& Johnson, Kymab, Leo Pharma, Medac, MSD, Neubourg Skin Care GmbH, Novartis, Pfizer, Principia Biopharma, Regeneron Pharmaceutical, Sandoz Biopharmaceuticals, Sanofi-Aventis, Sienna Biopharmaceuticals, Takeda, Trevi Therapeutics, UCB Pharma, Vascular Biogenics.

Katharina Drerup and Claudia Seemann have no conflicts of interest to disclose.

\section{References}

1 Blauvelt A, Papp KA, Griffiths CE, Randazzo B, Wasfi Y, Shen YK, et al. Efficacy and safety of guselkumab, an anti-interleukin-23 monoclonal antibody, compared with adalimumab for the continuous treatment of patients with moderate to severe psoriasis: Results from the phase III, double-blinded, placebo- and active comparator-controlled VOYAGE 1 trial. J Am Acad Dermatol. 2017;76(3):405-17.

2 Reich K, Armstrong AW, Foley P, Song M, Wasfi Y, Randazzo B, et al. Efficacy and safety of guselkumab, an anti-interleukin-23 monoclonal antibody, compared with adalimumab for the treatment of patients with moderate to severe psoriasis with randomized withdrawal and retreatment: Results from the phase III, double-blind, placebo- and active comparator-controlled VOYAGE 2 trial. J Am Acad Dermatol. 2017;76(3):418-31.

\section{Funding Sources}

No funding was received for this study.

\section{Author Contributions}

Katharina A. Drerup contributed through data acquisition, analysis, and interpretation, revising the work critically and approved the final version to be published.

Claudia Seemann contributed through data acquisition, analysis, and interpretation, revising the work critically and approved the final version to be published.

Sascha Gerdes contributed through the conception of the work, data acquisition, analysis, and interpretation, revising the work critically and approved the final version to be published.

Ulrich Mrowietz contributed through conception and design of the work, data acquisition, analysis, and interpretation, drafting the work and approved the final version to be published.

\section{Data Availability Statement}

All data generated or analyzed during this study are included in this article. Further enquiries can be directed to the corresponding author.
3 Gordon KB, Strober B, Lebwohl M, Augustin M, Blauvelt A, Poulin Y, et al. Efficacy and safety of risankizumab in moderate-to-severe plaque psoriasis (UltIMMa-1 and UltIMMa-2): results from two double-blind, randomised, placebo-controlled and ustekinumab-controlled phase 3 trials. Lancet. 2018; 392(10148):650-61.

4 Reich K, Papp KA, Blauvelt A, Tyring SK, Sinclair R, Thaci D, et al. Tildrakizumab versus placebo or etanercept for chronic plaque psoriasis (reSURFACE 1 and reSURFACE 2): results from two randomised controlled, phase 3 trials. Lancet. 2017;390(10091):276-88.
5 Masson Regnault M, Castaneda-Sanabria J, Diep Tran MHT, Beylot-Barry M, Bachelez $\mathrm{H}$, Beneton N, et al. Users of biologics in clinical practice: would they be eligible for phase III clinical studies? Cohort Study in the French Psoriasis Registry PSOBIOTEQ. J Eur Acad Dermatol Venereol. 2020;34(2):293300

6 Seneschal J, Lacour JP, Bewley A, Faurby M, Paul C, Pellacani G, et al. A multinational, prospective, observational study to estimate complete skin clearance in patients with moderate-to-severe plaque PSOriasis treated with BIOlogics in a REAL world setting (PSOBIO-REAL). J Eur Acad Dermatol Venereol. 2020;34(11):2566-73. 